\title{
Papers
}

\section{Exchanging assets and metadata across platforms}

\section{Jennifer Binder}

Since 2000, Jennifer has been involved in the development of digital asset management systems and services. She is the founder of Secure Digital Assets (SDA), a DAM company serving Fortune 1000 companies. SDA develops digital asset management systems (DAM) that archive and manage photos, graphics, audio and video. A central location where employees may Ingest, Search, Re-Purpose and Retrieve Digital Assets they need from any desktop 24/7. SDA's services enable a company to create integrated content that delivers a clear message consistent with the company's brand.

Keywords: metadata, SOAP, REST, HTTP, XML, assets, XMP, embed, export, import, multiple platforms

Abstract Software is growing as a service leading clients to choose providers based on UI, features and relationships instead of platforms. This is leading to a greater demand for systems to exchange assets on differing platforms. This article focuses on how an asset travels with metadata embedded in the file from one system to another. Journal of Digital Asset Management (2006) 2, 215-218. doi:10.1057/palgrave.dam.3650045

\section{INTRODUCTION}

As the demand to make workflows more efficient through digital processes grows, two main factors have opened the door to dramatically change the landscape. The ability to easily embed data into an asset at multiple points of the workflow and the ability to exchange assets across platforms have revolutionized workflows. Gathering accurate metadata at the earliest stage by the content creator is simple with the use of Extensible Metadata Platform (XMP). Once the asset moves into a managed digital asset management (DAM) system, a director may edit and embed new data into the file. Once the asset has been posted, it may travel safely across platforms into other systems with the insurance that its data will be there to describe what the asset is and how it can and cannot be used.

\section{CAPTURING METADATA IN ASSETS USING XMP}

The earlier metadata are embedded digitally into

Jennifer Binder CEO, SD Assets, 101 West 23rd St. New York, NY 10011, USA Email: jennifer@sdassets. com an asset, the better, and it can start with the content creator using XMP. XMP is an Adobe product that is compatible with all Adobe applications. XMP allows for descriptive information to be entered into standardized fields where creators can add a title, author, description, keywords, copyright notice, headline, instructions and more. As soon as these data are entered into an asset, it becomes a part of that asset and travels with it. XMP automatically picks up the file's properties like date created, format, resolution, history and more. This is a win-win for all, the content creator is guaranteed credit and explanation of proper copyright usage and the client receives metadata formatted in the file from the get go. This translates into less work for the client and more security for the content creator.

\section{IMPORTING ASSETS INTO A DAM SYSTEM AND AUTO-POPULATING FIELDS IN THAT SYSTEM}

If metadata has been entered into an asset's XMP fields and the DAM system has existing fields that are designed to recognize XMP, then an asset can automatically populate descriptive fields of a DAM cutting down the amount of data a director/ manager needs to input. This sounds as if it is just cutting out one step of the workflow but it is actually much more than that. If an asset does not have any metadata associated with it and the director/manager does not know the information, 
then it could take a great deal of research to track down the author, copyright, keywords and description, if it could be recovered at all. Fields within a DAM system can be mapped to match up with standard XMP fields. Minimizing the amount of research needed to input metadata for an asset expedites populating a DAM and allows directors/managers to use a DAM with greater freedom enhancing the workflow and allowing directors to focus on core business objectives.

\section{USING WEB SERVICES TO EXPORT AND EXCHANGE ASSETS FROM ONE SYSTEM TO ANOTHER}

Web services create a method to provide and move data across platforms. There are a host of web services to exchange data across ODBC, JDBC, ADO and ADO.Net platforms. For media rich content, the best two web services

XMP Mapping

\begin{tabular}{|c|c|c|c|c|}
\hline Action & Taq Name & \multicolumn{3}{|c|}{ Field Association } \\
\hline Edit I Delete & Image Copyright & \multicolumn{3}{|c|}{ Copyright } \\
\hline Edit I Delete & Image Description & \multicolumn{3}{|c|}{ Description } \\
\hline Edit I Delete & Image Author & \multicolumn{3}{|c|}{ Author } \\
\hline \multicolumn{5}{|l|}{ New Mapping } \\
\hline XMP Tag Name: & Image Owner & \multirow[t]{2}{*}{ Field Association: } & Copyright Owner & $\checkmark$ \\
\hline Save Cancel & & & & \\
\hline
\end{tabular}

\section{EDITING AND EMBEDDING DATA IN A DAM}

Flexibility to modify XMP data within a DAM is critical. A DAM must be able to ingest XMP data then embed new data to a single asset or group of assets. Ideally, a DAM will allow directors/ managers to assign data to a group of assets and edit data of a single asset that will be embedded into the file and travel with the assets once it leaves its native DAM system. Without the ability to edit and embed, metadata are held hostage once it is in the system. Creating standard fields to recognize and update metadata in systems allows for more assets to get into a DAM increasing the value of the assets and making them more available to a larger audience. that create interoperability among different environments are Simple Object Access Protocol (SOAP) and Representational State Transfer (REST). Interoperability is key because it allows objects and code of any kind from any platform in any language to communicate with another platform.

SOAP and REST create a glue that binds systems together allowing data and assets to cross over. Systems no longer need to run on the same platform or be written in the same language. SOAP and REST are both based on XML and use HTTP to communicate. When choosing between SOAP and REST, it is best to evaluate what the objectives are and select the best method. Generally, REST is faster, easier to

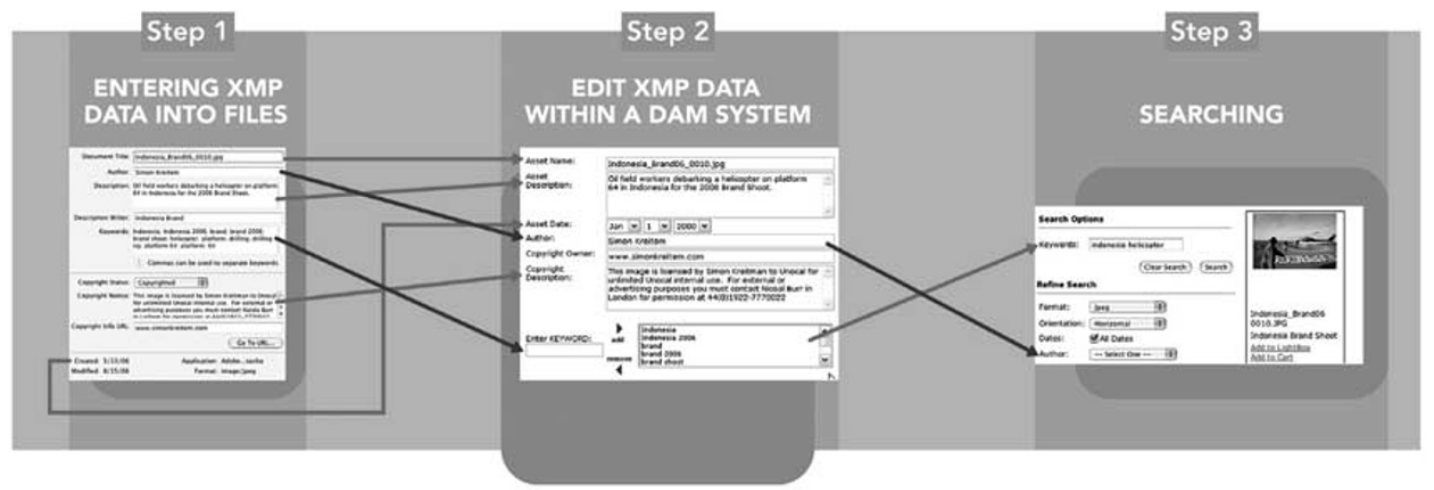


build and maintain and does not require an additional toolkit. REST is lightweight without a lot of additional XML mark-up with human readable results. SOAP communicates exceptions in a standardized manner so there is no need for proprietary exception communication mechanism. SOAP can be easier to consume with no worries about payload. SOAP and REST both resolve the conflict between COM and JAVA component object models and create a neutral method of exchange for interoperability. populate fields of a DAM. Some XMP fields like keywords, descriptions and authors can become searchable in a DAM greatly reducing the amount of input an administration has to make per batch of assets or individual asset. XMP organizes data in an asset that can be ingested into a DAM creating searchable content and reducing the time needed to manage the system.

Protecting metadata associated with an asset is the key to better re-usage of the asset. DAMs increase the value of assets when more users

\section{Secure Digital Assets REST Data Exchange}
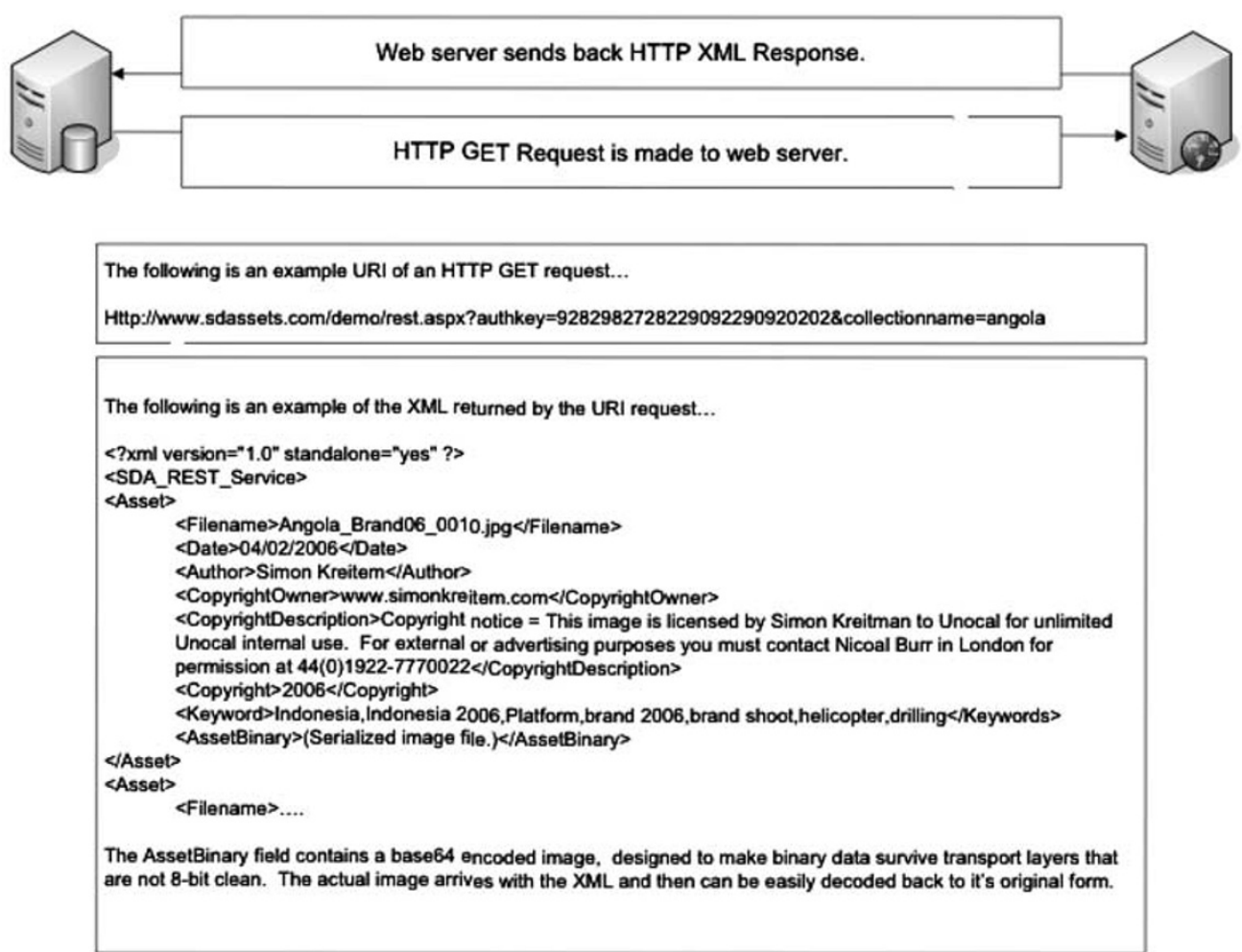

\section{RESULTS: ENHANCED WORK FLOW AND INSURED PROTECTION OF METADATA}

When managing a DAM, it can be overwhelming to consider importing hundreds of thousands of assets and describing batches of them or each one individually over time. With XMP, the data are already a part of a digital asset with key descriptive fields that may auto- know where the assets are and how to use them. When the metadata of an asset is embedded in the file using XMP then imported into a system where it can be viewed and edited then exported out with the asset, the asset is in essence insured to communicate what it is and how it can be used wherever it lands. The choice of using and describing the asset properly becomes the responsibility of the end user. 
With the growing use of XMP, it is now safe to share assets between systems and with the advances of SOAP and REST it is much easier for systems to effectively exchange assets across COM and JAVA platforms. As the flexibility of exchanging data across platforms increases, clients may make decisions on UI, functionality and relationships instead of platform-specific applications. It is always best to choose a software provider that understands your industry and needs. For every service, there is a web-based application to support the service whether it is in terms of distribution, creation, communication, sales and/or organization. Service-oriented architecture opens the door for you to choose vendors that understand your business and needs. Interoperability allows for data to flow quickly and seamlessly across the internet in managed systems. 